\title{
The Association Between Hypermobility Ehlers-Danlos Syndrome and Other Rheumatologic Diseases
}

\author{
Authors: $\quad$ *AK Makol, $\quad$ $\quad$ B Chakravorty, ${ }^{1}$ MB Heller, ${ }^{2}$ B Riley B ${ }^{2,3}$ \\ 1. New York Institute of Technology College of Osteopathic Medicine, Long Island, \\ USA \\ 2. Department of Family Medicine, New York Institute of Technology College of \\ Osteopathic Medicine, Long Island, New York, USA \\ 3. Ehlers-Danlos Syndrome / Hypermobility Treatment Center, New York Institute of \\ Technology College of Osteopathic Medicine, Long Island, New York, USA \\ Disclosure: $\quad$ The authors have declared no conflicts of interest. \\ Received: $\quad 05.04 .21$ \\ Accepted: $\quad 26.08 .21$ \\ Keywords: $\quad$ Ankylosing spondylitis (AS), hypermobility Ehlers-Danlos syndrome (hEDS), \\ rheumatoid arthritis (RA). \\ Citation: $\quad$ EMJ. 2021; DOI/10.33590/emj/21-00078R2
}

\begin{abstract}
Research has shown hypermobility Ehlers-Danlos syndrome (hEDS) to be associated with some complicated rheumatologic disease. In this feature paper, the authors discuss the prevalence and pathophysiology of rheumatologic conditions, specifically ankylosing spondylitis and rheumatoid arthritis, in patients with hEDS. Furthermore, the authors discuss possible reasons for the association of hEDS with these rheumatologic diseases.
\end{abstract}

\section{INTRODUCTION}

Research has shown that hypermobility EhlersDanlos syndrome (hEDS) is associated with rheumatologic disease.' In this article, the authors first discuss the clinical features and pathogenesis of hEDS, rheumatoid arthritis (RA), and ankylosing spondylitis (AS). They examine the association between hEDS and rheumatological disease closely by discussing the prevalence of RA and AS in patients with hEDS as compared to the general population of the USA. In addition, they explore possible causes for this association.

\section{CLINICAL FEATURES AND} PATHOGENESIS OF HYPERMOBILITY EHLERS-DANLOS SYNDROME

A heritable connective tissue disorder, hEDS is characterised by joint hypermobility, musculoskeletal, skin, and soft tissue symptoms. ${ }^{2}$ The clinical course of hEDS follows three distinct phases: a 'hypermobility phase', a 'pain phase', and a 'stiffness phase'. The 'hypermobile phase' occurs early in life and increases risk for joint sprains and dislocations. ${ }^{2}$ Patients in this phase often experience pain predominantly in the lower limbs and easy fatigability. Patients begin to experience the 'pain phase' in their 20s-40s. In this phase, patients experience worsening musculoskeletal pain, paresthesias, and 
gastrointestinal disorders. ${ }^{2}$ The 'stiffness phase' is characterised by pain and reduction of joint mobility to a debilitating degree. ${ }^{2}$ hEDS is also multisystemic in nature. Patients often struggle with a plethora of non-musculoskeletal symptoms such as dysautonomia, chronic fatigue, abnormal proprioception, gastrointestinal dysmotility, and mood disorders. ${ }^{3}$

\section{Pathogenesis}

There is no conclusive evidence confirming the aetiology of hEDS. However, transcriptome profiling of hEDS cells revealed a transcriptional change leading to "fibroblast-to-myofibroblast transition." 4 This transition results in cells with an increased ability for contraction, which can explain some systemic manifestations of hEDS such as gastrointestinal dysfunction, chronic musculoskeletal pain, and soft tissue inflammation. ${ }^{4} \quad$ Furthermore, continuous activation of myofibroblasts leads to impaired wound healing, thus explaining the soft tissue lesions patients with hEDS experience. ${ }^{4}$ Transcriptome profiling of hEDS cells also revealed a dysfunctional signalling pathway between TGF- $\beta$ and Wnt, resulting in continuous post-inflammatory fibrosis and myofibroblast formation. ${ }^{4}$ Though the specific molecular mechanism causing chronic pain in patients with hEDS is not known, it is presumed that "inflammation-related genes" such as spondin-2 are upregulated. ${ }^{4}$ Spondin-2 codes for many functions of the innate immune system, and is also involved in inflammatory cell recruitment. ${ }^{4}$ This dysregulation is believed to increase painful sensations in patients with hEDS. ${ }^{4}$

\section{CLINICAL FEATURES AND PATHOGENESIS OF RHEUMATOID ARTHRITIS}

RA is an inflammatory disease that attacks the joints. ${ }^{5}$ Continuous inflammation in the synovium leads to damage in the affected joints, causing pain and functional deterioration in patients. ${ }^{5}$ RA typically starts off insidiously with fatigue and generalised muscle pain, and progresses within weeks to months to involve the joints in a symmetric pattern. ${ }^{6}$ The most commonly affected joints are in the hands, feet, wrists, ankles, elbows, and knees. ${ }^{6}$ These joints tend to appear swollen, warm, and are particularly stiff and painful in the morning or following a period of inactivity. ${ }^{6}$ As RA can lead to inflammation of surrounding tendons, ligaments, and skeletal muscle, patients can experience radial deviation of the wrist, ulnar deviation of the fingers, swanneck, and boutonniere deformities of the fingers. ${ }^{6}$ Joints affected with RA will have a minimal range of movement in a waxing and waning course. ${ }^{6}$

\section{Pathogenesis}

A combination of genetic and environmental factors lead to a loss of self-tolerance to a native protein that contains a citrullinated residue. ${ }^{5}$ Due to this lack of tolerance, the body then develops antibodies against these citrullinated residues, called anti-citrullinated peptide antibodies. ${ }^{5}$ Both the adaptive and innate immune system are involved in an inflammatory reaction causing leukocytes to enter into the synovium and cause joint destruction. ${ }^{5}$ The inflammatory response is persistently activated in patients with RA, causing continuous progression of the disease.

\section{CLINICAL FEATURES AND PATHOGENESIS OF ANKYLOSING SPONDYLITIS}

AS is an inflammatory disease that causes the destruction of articular cartilage of the sacroiliac and apophyseal joints, resulting in bony ankylosis. ${ }^{6}$ The classic symptoms of AS include inflammatory back pain that is worse in the morning or during periods of inactivity, which persists for longer than 3 months and improves with movement. ${ }^{7}$ Additionally, patients with AS experience reduced spinal mobility later in the advanced stage of the disease. ${ }^{7}$ The progression of the disease can be followed and distinguished in radiologic imaging. Early imaging results will reveal reactive sclerosis and syndesmophyte formation at the edges of the vertebral bodies. ${ }^{7}$ Late imaging results will show a "bamboo spine" resulting from bony bridging. ${ }^{7}$

\section{Pathogenesis}

The pathogenesis of AS is poorly understood. It is predicted that enthesitis, which is defined as "the insertion of a tendon, ligament, capsule, or fascia into bone," is the major hallmark of AS. ${ }^{8}$ Recent studies show that the immune system attacks the enthesis of the intervertebral 
discs and the annulus fibrosus, which contains fibrocartilage as well. ${ }^{8}$ Patients with AS were found to have a higher concentration of cluster of differentiation-8+ T-cells, leading to the possible theory that T-cells from the bone marrow invade the fibrocartilage. ${ }^{8}$ Some studies have shown that antigens from fibrocartilage presented by human leukocyte antigen-B27 (HLA-B27) to cluster of differentiation-8+ T-cells is the pathologic basis for AS. $^{8}$

\section{PREVALENCE OF RHEUMATOID ARTHRITIS AND ANKYLOSING SPONDYLITIS IN HYPERMOBILITY EHLERS-DANLOS SYNDROME}

A study conducted in 2017 showed that hEDS is associated with rheumatologic conditions. This study examined the number of patients with hEDS who tested positive for HLA-B27, a characteristic feature heavily correlated with AS. 1.8 Of the patients with hEDS who received a complete serological and radiographic workup, 24\% tested positive for HLA-B27. In comparison, the prevalence of HLA-B27 in the general population of the USA is $6.1 \% .^{1}$ Additionally, in this study, $6.8 \%$ of patients with hEDs were also diagnosed with RA. ${ }^{1}$ Comparatively, the prevalence of RA between 2004-2014 in adults in the USA ranged from $0.41-0.54 \% .^{9}$ As shown in the studies conducted by Rodgers et al. ${ }^{1}$ and Hunter et al., 9 patients with hEDS have a higher prevalence of RA and characteristic genetic marker for AS.

Compared to HLA-B27, 3.4\% of hEDS patients who received a complete serological and radiographic workup tested positive for anticitrullinated protein antibody. In comparison, a cohort study showed that the percentage of anticitrullinated protein antibody positivity in the general population is $1.0 \%{ }^{10}$

\section{PLAUSIBLE REASONS FOR ASSOCIATION}

Rheumatological conditions such as RA and AS are associated with hEDS.' However, because the aetiology of hEDS is largely unknown, the understanding for this association remains limited.1,4 The pathogenesis of hEDS is thought to include an increased fibroblast-to-myofibroblast transition, along with a dysfunctional signalling pathway leading to post-inflammatory fibrosis. ${ }^{4}$ In comparison, RA contains an autoimmune response to a decreased self-tolerance capability of T-cells. ${ }^{5}$ In addition, studies have predicted that the pathogenesis of AS is related to the dysfunction between HLA-B27 signalling to T-cells, causing an inflammatory response in fibrocartilage. ${ }^{8}$ Due to the apparent differences in mechanisms of pathogenesis in RA and AS when compared to hEDS, it is difficult to specify the cause of association.' However, the authors think since the basis of hEDS lies in a dysregulation of the "inflammation-related genes," 4 patients with hEDS are more susceptible to developing autoimmune conditions, such as RA and AS.

\section{LIMITATIONS}

In order to fully understand the association between hEDS, RA, and AS the genetic basis of hEDS is an important factor; however, it is beyond the scope of this review. Although clinically these conditions may present similarly, further studies have to be conducted in order to establish a concrete reasoning for the association between hEDS and rheumatological conditions. In particular, the authors suggest the need for future research to explore the molecular mechanisms of hEDS, which may lead to its association with other rheumatologic diseases. There has been limited qualitative research done to support the association between hEDS, RA, and AS and, therefore, a concrete connection between these conditions is difficult to establish. Additionally, it should be noted the pathophysiology of RA and AS are more comprehensive than what is included. Only the pathophysiology that is relevant for comparing RA and AS to hEDS has been presented. 


\section{References}

1. Rodgers KR et al. Ehlers-Danlos syndrome hypermobility type is associated with rheumatic diseases. Sci Rep. 2017;7:39636.

2. Tinkle B et al. Hypermobile EhlersDanlos syndrome (a.k.a. EhlersDanlos syndrome type III and EhlersDanlos syndrome hypermobility type): clinical description and natural history. Am J Med Genet C Semin Med Genet. 2017;175(1):48-69.

3. Riley B. The many facets of hypermobile Ehlers-Danlos syndrome. J Am Osteopath Assoc. 2020;120(1):30-2.
4. Chiarelli $\mathrm{N}$ et al. Cellular and molecular mechanisms in the pathogenesis of classical, vascular and hypermobile Ehlers-Danlos syndromes. Genes (Basel). 2019;10(8):609.

5. Gibofsky A. Epidemiology, pathophysiology, and diagnosis of rheumatoid arthritis: a synopsis. Am J Manag Care. 2014;20(Suppl 7);128-35.

6. Robbins SL et al. Pathologic Basis of Disease (2020). Philadelphia, PA: Saunders Elsevier.

7. Golder V, Schachna L. Ankylosing spondylitis: an update. Aust Fam
Physician. 2013;42(11):780-4.

8. Sieper J et al. Ankylosing spondylitis: an overview. Ann Rheum Dis. 2002;61(Suppl 3):iii8-18.

9. Hunter TM et al. Prevalence of rheumatoid arthritis in the United States adult population in healthcare claims databases, 2004-2014. Rheumatol Int. 2017;37(9):1551-7.

10. van Zanten A et al. Presence of anticitrullinated protein antibodies in a large population-based cohort from the Netherlands. Ann Rheum Dis. 2017;76(7):1184-90. 